Noname manuscript No.

(will be inserted by the editor)

\title{
The Abell cluster A586 and the detection of violation of the Equivalence Principle
}

\author{
Orfeu Bertolami · Francisco. Gil Pedro • \\ Morgan Le Delliou
}

Received: 18/12/08 / Accepted: date

\begin{abstract}
We discuss the current bounds on the Equivalence Principle, in particular from structure formation and, reexamine in this context, the recent claim on the evidence of the interaction between dark matter and dark energy in the Abell Cluster A586 and the ensued violation of the Equivalence Principle.
\end{abstract}

Keywords Cosmology · Gravitation · Dark Matter · Equivalence Principle · Field-theory

PACS 98.80.-k $\cdot 98.80 . \mathrm{Cq}$

\section{Introduction}

The Equivalence Principle (EP) is a basic physical ingredient of general relativity and shown to hold to great accuracy in composition dependent free fall experiments involving known forms of matter. However, recent discovery of empirical evidence of the existence of dark matter (DM) in the cluster of galaxies $1 E 0657$ - 56, the so-called "bullet cluster" [1, and in a ring-like DM structure in the core of the galaxy cluster $C l 0024+17$ 2], together with proposals on coupled dark energy (DE) have opened up the possibility of having

Preprint: DF/IST-4.2007

O. Bertolami and F. Gil Pedro

Departamento de Física, Instituto Superior Técnico

Av. Rovisco Pais 1, 1049-001 Lisboa, Portugal

E-mail: orfeu@cosmos.ist.utl.pt and fgpedro@fisica.ist.utl.pt

O.B. Also at: Centro de Física dos Plasmas, IST.

M. Le Delliou

Instituto de Fsica Terica UAM/CSIC, Facultad de Ciencias, C-XI, Universidad Autónoma de Madrid, Cantoblanco, 28049 Madrid SPAIN E-mail: morgan.ledelliou@uam.es

Also at: Centro de Física Teórica e Computacional, Universidade de Lisboa, Av. Gama

Pinto 2, 1649-003 Lisboa, Portugal, delliou@cii.fc.ul.pt 
dark sector interactions which could alter the free fall of dark matter (DM) and DE as compared to baryons.

In a recent paper 3, we have argued that the study of the virial equilibrium of the cluster Abell A586 indicates DE-DM interaction and that this induces a detectable violation of the EP. This prompted a discussion on the connection with existing related work on the free fall in the presence of DM [4,6] as well as on the phenomenological bounds on the interaction of DE and DM arising from cosmology [7].

In this paper we examine the consistency of the results of Bertolami et al. 3] with the existing literature. Our discussion is organized as follows: in section 2 we briefly summarize the way EP violations can be detected. We then compare our previous results with homogeneous cosmology data in section 3 and express them in terms of free fall in structure formation in section 4 Section 5 contains our conclusions.

\section{An EP Violation Typology}

One can identify two qualitatively distinct forms of violation of the EP: i) A more classic one, where one observes a differential acceleration on the free fall of baryons in a gravitational field, the so-called fifth force type experiments and ii) A dark sector violation, where one compares the free fall of baryons with the one of DM or DE in a cosmological setting.

\subsection{Baryonic composition dependent violation of the EP}

In the fifth force type experiments one is concerned with the detection of the difference on the free fall acceleration of baryonic matter with different composition. A putative difference would be due to a composition dependent new interaction. Current bounds on such a differential acceleration imply that it should be smaller than $0.3 \times 10^{-13}[8$. Alternatively, one could consider a differential gravitational acceleration for baryonic matter and for DM or DE (DM and DE if these two entities are interacting). The situation with DMbaryons composition dependent interaction was examined in Stubbs 4 and the bound $3.7 \times 10^{-3}$ for the differential acceleration of galactic DM on different baryonic test masses was obtained. The latest bound however is $(-4 \pm 7) \times$

$10^{-5}$ 8. For sure, existing laboratory tests of the EP are irrelevant in what concerns its validity on the dark sector. For a general discussion of the EP validity for known particles and antiparticles, see, for instance, Bertolami et al. 9.

\subsection{Dark sector violation of the EP}

The fact that recent observations suggest that about $96 \%$ of the content of the universe is composed by dark material of yet unknown nature opens up the 
possibility of considering models where the free fall of dark sector particles is different from the one of the baryonic sector. Since the EP relies on clustering of mass, a violation of the EP would be detected by comparing the baryonic free fall with the clustering component of the dark sector: DM. That entails the two possibilities for such modification: either the self-gravity of DM gets modified in the particular fashion suggested in Kesden and Kamionkowski [5], or this modification is induced by an additional interaction between clustered and unclustered components of the dark sector, DM and DE, respectively [3. Contrary to the previous case, detection cannot rely on free fall experiments of different baryonic materials, but must involve baryon and DM falling onto DM [5, 3].

Such configuration involves the formation of large scale structure, at ranges where DM plays a leading role. In Bertolami et al. [3], we have used the gravitational relaxation in a galaxy cluster, A586, and its peculiar type of statistical equipartition of energy to test for the presence of modifications with respect to the dust behaviour globally assigned to clustering matter. We have detected such modification and identified it with the DE-DM interaction, which itself logically entails a violation of the EP. We have proposed in Bertolami et al. 3 that such violation should be detectable in a generic bias present at the homogeneous level. In section 4, we show that a particular model of DE-DM interaction yields a variation of the gravitational coupling equivalent to the effects of the ad hoc model considered in Kesden and Kamionkowski [5. Such variation is used there to separate the free fall of baryons (stars) onto the DM filled galactic centre at a different rate than that of DM sub-haloes of the Milky Way.

\section{DE-DM Interaction and EP Violation}

Models of interactions in the dark sector have an impact on the EP since they modify the conservation of DM, at least at the homogeneous level. Such modification entails detectability on the evolution of the homogeneous geometry of the universe, as shown for the phenomenologically consistent unified model of DE and DM [10, the generalized Chaplygin gas (GCG) model [11] or for the more generic interacting model [7,12, but also on the quality of the self-gravitating equilibrium reached by large amounts of DM in clusters [3]. In Guo et al. [7, the consistency of two DE-DM interaction models, a constant coupling model and a varying coupling model [13], with respect to the conjunction of data from Cosmic Microwave Background (CMB) radiation shift parameter [14, SNLS observations of type Ia Supernovae (SNIa) [15] and the Baryon Acoustic Oscillation (BAO) peaks in the SDSS 16. The varying coupling model was also considered, together with the GCG model, in the study of the A586 cluster [3, in which detection of the DE-DM interaction was inferred from departure of the virial equilibrium. In what concerns the varying coupling model, observational evidence favors the detection of interaction in 


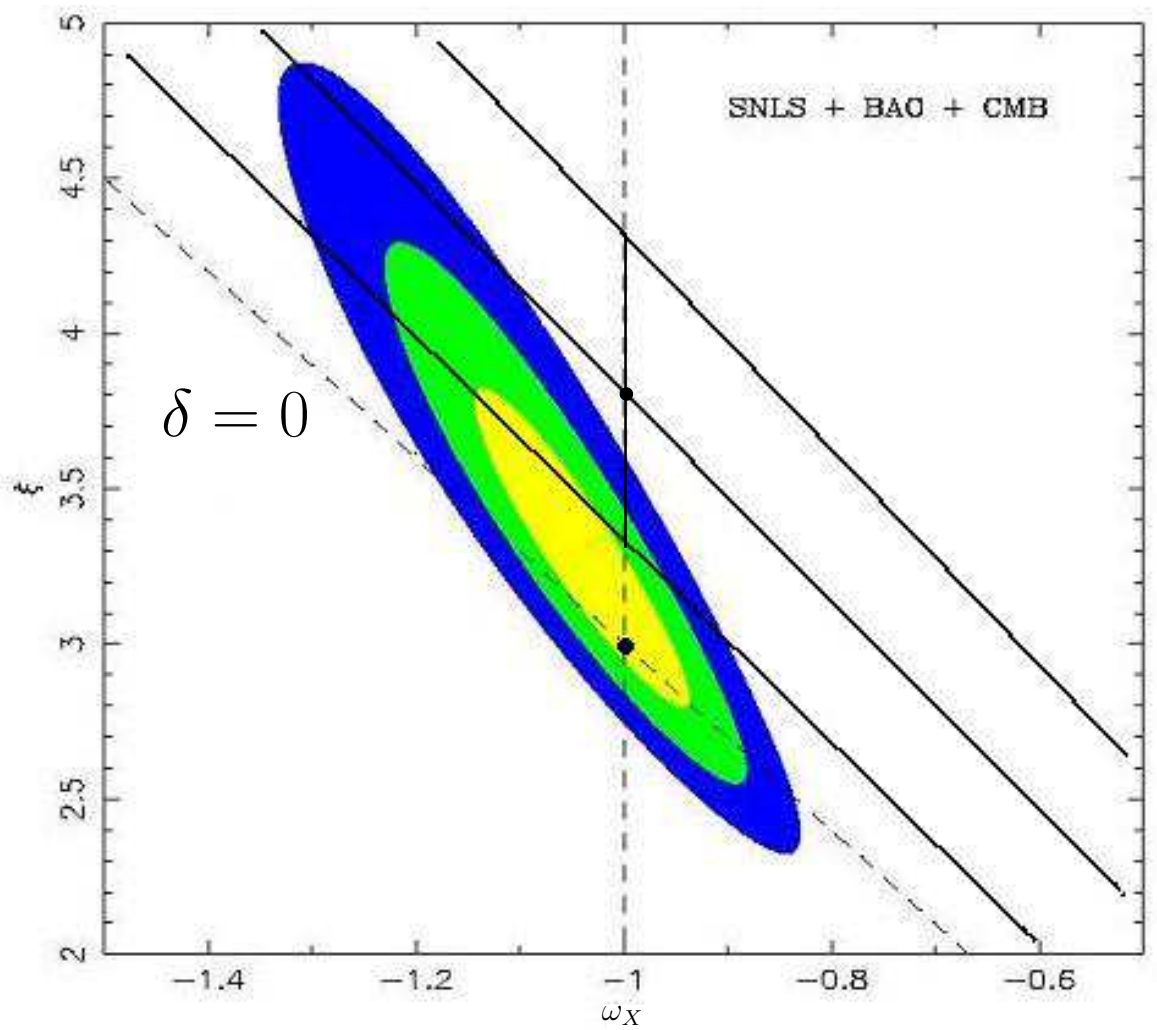

Fig. 1 Superimposition of the probability contours for the interacting DE-DM model in the $\left(\omega_{X}, \xi\right)$ plane (denoted as $\left(\omega_{D E_{0}}, \eta\right)$ in Bertolami et al. [3]), marginalized over $\Omega_{D E_{0}}$ in Guo et al. [7] study of CMB, SNIa and BAO $(2.66<\xi<4.05$ at $95 \%$ C.L.) with the extended results of Bertolami et al. [3] based on the study of A586 cluster. The $\xi=-3 \omega_{X}$ dashed line corresponds to uncoupled models. The result of Bertolami et al. $\left[3\right.$ is set at $\omega_{X}=-1$ and error bars are shown. The thick lines corresponds to their extension to varying $\omega_{X}$.

the region where both approaches are compatible, namely for $\omega_{X}<-1$ (see Fig. 11).

The absence of detection in the homogeneous study [7] at $1 \sigma$ level can be explained by the redshifts of CMB, SNIa and BAO, which are much larger than the one of A586, while in the regime of dominance of DE, the latter object is more affected. Should we have access to more systems like A586, an error analysis would be possible, adding the virial equilibrium as a Gaussian prior to obtain a joint likelihood analysis with the homogeneous constraints. With only one system to analyze, the signature shown on Fig. 1 1 merely superimposes the results from Guo et al. 7 ] with our study [3] with the respective errors. The overlap of contours from both studies favors interacting phantom-type models $\left(\omega_{X}<-1\right)$ with energy transfer from DM to DE, as found in Bento et al.and Majerotto et al. [17, 18]. 


\section{EP Violation, Free Fall and Structure Formation}

In their simulation of a particular modification of gravity for DM, Kesden and Kamionkowski [5 conclude that the remnants of stars (baryonic matter) in the heading tidal streams of the Sagittarius Milky Way satellite limits the variations induced by the resulting variation of gravitational coupling, $G$, up to $10 \%$. Considering a model for the DE-DM interaction together with constant mass DM particles, the effect on free fall gravitational force is also to modify the coupling with time: the DM particle number density, $n$, is diluted due to expansion and due to interaction with DE. This interaction can be modeled as an extra acceleration $a_{\text {Int }}$. felt by DM particles on top of $a_{\text {grav }}$., the gravity of the cluster. Since this extra acceleration is only felt by DM particles, the violation of the EP is explicit in this model. Furthermore, using that the relative change of total acceleration caused by this interaction is due to the relative change in particle number density with respect to expansion over a given cosmological time interval $\Delta t$ :

$$
\frac{a_{\text {Int. }}}{a_{\text {grav. }}} \propto \frac{\left(\dot{n}_{D M}-\dot{n}_{D M} \|_{\text {expansion }}\right) \Delta t}{n_{D M}} .
$$

Since this is a cosmological effect, it is natural to consider $\Delta t \propto 1 / H$, the Hubble time. The constant DM mass hypothesis leads to $\dot{\rho}_{D M}=\dot{n}_{D M} m_{D M}$, which from the Bianchi identity, Eq. (1) from Bertolami et al. [3], turns into

$$
\dot{n}_{D M}+3 H n_{D M}=\zeta H n_{D M},
$$

where

$$
\zeta=-\frac{\left(\eta+3 \omega_{D E}\right) \Omega_{D E_{0}}}{\Omega_{D E_{0}}+\Omega_{D M_{0}} a^{-\eta}} .
$$

Now, absence of interaction $(\zeta=0)$, Eq.(2) would yield $\dot{n}_{D M} \|_{\text {expansion }}=$ $-3 H n_{D M}$. Thus, introducing a phenomenological constant $\delta$ that also absorbs the Hubble time fraction, and using Eq. (2), one obtains

$$
\frac{a_{\text {Int. }}}{a_{\text {grav. }}}=\delta \frac{\dot{n}_{D M}-\dot{n}_{D M} \|_{\text {expansion }}}{n_{D M} H}=\delta \zeta .
$$

Since this ratio depends only on the scale factor, $a$, the relative change in acceleration is conveyed directly to the effective DM gravitational coupling, $G_{D M}$ :

$$
G_{D M}=G(1+\delta \zeta),
$$

which allows for a straightforward comparison with the simulation of Kesden and Kamionkowski [5. As this change is due to the interaction with DE, which is significant only quite recently in the history of the universe, $G_{D M}$ decreases as the strength of interaction becomes more important. Notice that the interaction vanishes in the past and then $G_{D M}(z)=G$. Given that the

study of Kesden and Kamionkowski [5] sets from the observations a limit of about $\varepsilon=10 \%$ as the tolerance for such a change, we choose it to be the value 
for $G_{D M}$ at present, therefore in the present model $\delta=\varepsilon /|\zeta|=0.163$, assuming that $\omega_{D E_{0}}=-1, \Omega_{D E_{0}}=0.72, \Omega_{D M_{0}}=0.24$, from which follows that $\eta=3.82_{-0.47}^{+0.50}$. Notice that the negative sign, given by our observation of A586's virial ratio, arises from the fact that the detected energy transfer is from DM to DE, i.e. one has less DM and hence effectively a smaller effective gravitational coupling. For sure, one does not expect a negative overall coupling as the discussed effect is presumably small and ours is just a phenomenological model of the complex process of interaction between concentrations of DM, which are at the same time turning into DE. It is interesting to remark that as the flow of energy density is from DM to DE, then $n_{D M}$ is further decreased with respect to expansion, which translates into a decrease on the coupling of DM at present. Thus, one expects streams of stars to head the tidal arms of absorbed satellites in this model.

Notice also that this calculation explicitly aims to translate the interaction with DE into a phenomenological variation of gravity in clusters. Of course, the value of $\varepsilon$ is chosen conservatively so that the modification is compatible with the findings of Kesden and Kamionkowski [5. However, it is fair to say that only a simulation with varying $G_{D M}$ would be fully conclusive. Actually, it is quite conceivable that observations are consistent with even higher values of $\varepsilon$.

The behaviour of Eq. (5) is shown in Fig. 2.

In the following, we discuss some of the salient features of the proposed model. For the Milky Way satellite, the observed stream evolution runs through at least 4 orbits that can be evaluated at 0.85 Gyr each. This corresponds to redshifts between about $z=0.36$, where it started with $G_{D M} / G=0.94$, and $z=0$, with a midpoint in evolution time at $z=0.15$ where the ratio is 0.91 . For the A586, $z=0.17$, and one obtains changes of $G_{D M}$ of about $8 \%$ for the values of Bertolami et al. [3] (see above), as Eq. (5) yields $G_{D M} / G=0.92$. Notice that the observations of tidal streams from satellites of galaxies thus far should reveal the kind of trailing studied in Kesden and Kamionkowski [5]. [Furthermore, the negative contribution to the effective gravitational coupling can be attributed to the phantom-like nature of the interacting DE model.]

It is relevant to point out that, in the context of the bullet cluster $(z=$ 0.296), an analysis of the velocity of the "bullet" sub-cluster indicates that a long range force with strength $G_{D M} / G=1.4-2.2$ is needed to account for the rather high observed velocities [6], even though a more elaborate analysis [19] suggests that more detailed hydrodynamical models are required to reach a definite conclusion. Our fit (which yields $G_{D M} / G=0.93$, for $z=0.296$ with the values from Bertolami et al. [3]) does not account for such a large deviation from the Newtonian regime. And clearly, a $\delta>0$ is not even qualitatively consistent with it, however, we stress that the parameter $\delta$ reflects our

\footnotetext{
1 We point out that errors in Bertolami et al. 3] were underestimated. The correct results are as follows: the virial ratio $\frac{\rho_{K}}{\rho_{W}}=-0.76 \pm 0.14 \neq-0.50$, the $\omega_{D E}=-1$ interacting pseudo-quintessence scaling $\eta=3.82_{-0.46}^{+0.50} \neq-3 \omega_{D E}$ and the generalized Chaplygin gas power $\alpha=0.27_{-0.15}^{+0.17} \neq 0$. Conclusions remained unchanged.
} 


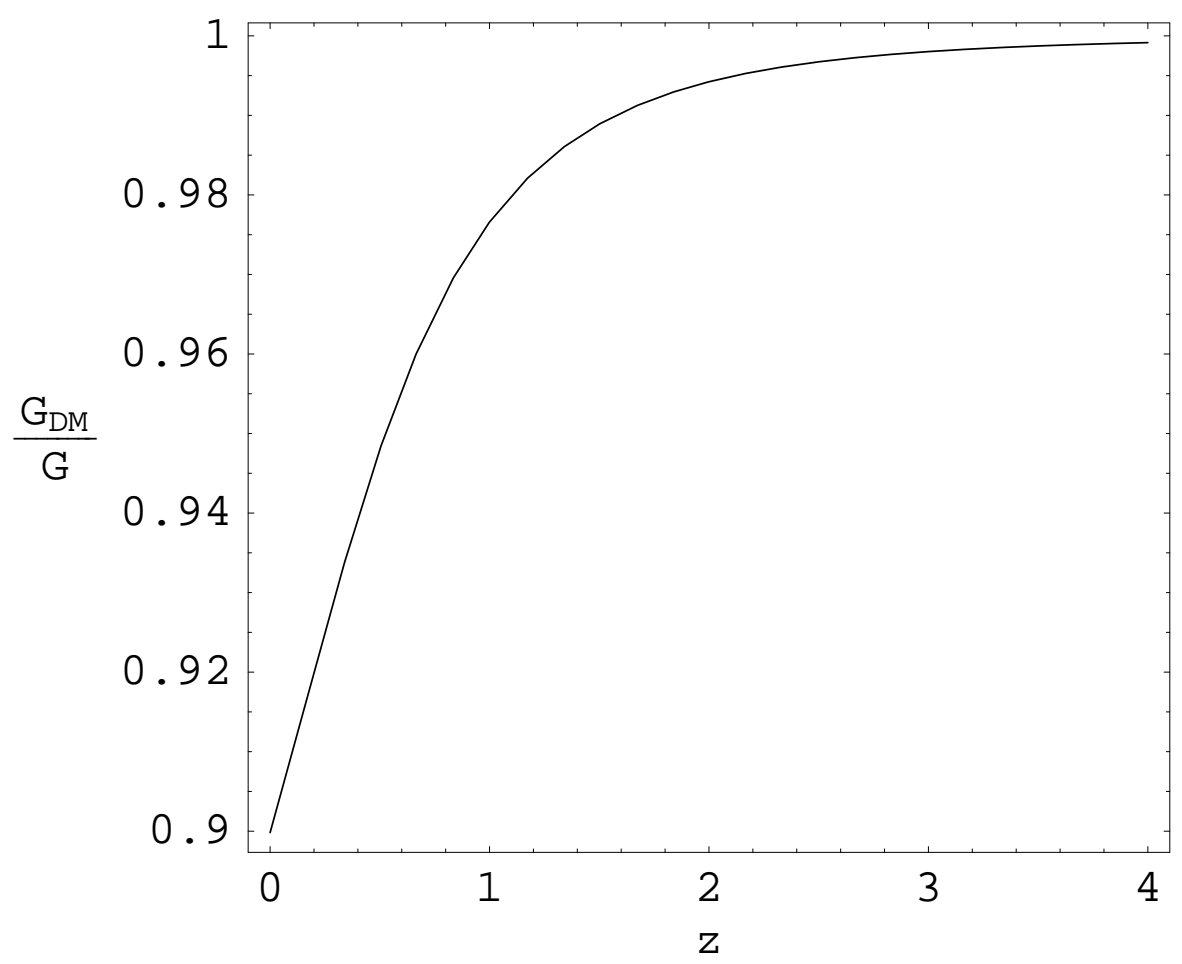

Fig. 2 Evolution with redshift of the ratio of the gravitational coupling for DM and baryons falling on a DM halo, using the varying coupling model discussed in Bertolami et al. [3], as compared with the simulation of Kesden and Kamionkowski [5].

ignorance on the correct model of the interaction process and its details. In this context, it is interesting to point out that if instead, $\delta$ were negative, then our approach could, at least in principle, be consistent with values required to accommodate the bullet cluster dynamics.

Finally, it is worth remarking that our results are consistent with limits on the variation of the gravitational coupling with the energy scale [22].

\section{Discussion and Conclusions}

In this paper we have examined the compatibility of the existing studies on the interaction of dark matter and dark energy and the results of Bertolami et al. 3] concerning the Abell A586 cluster. We have shown that there is no 
contradiction with the available cosmological data, at least in what concerns the varying coupling model of interaction between DE and DM. Furthermore, we find that if one interprets the evidence of EP violation from the Abell A586 as a change in the gravitational coupling, then our results match those obtained in Kesden and Kamionkowski [5] through a simulation of the tidal stream of stars in the Sagittarius dwarf galaxy due to DM. For sure, a more accurate comparison would involve considering a simulation carried out in Kesden and Kamionkowski [5] with the gravitational coupling varying according to Eq. (5). However, this is clearly beyond the scope of this work.

It should be mentioned that an attractive new force or a stronger gravitational coupling for DM might be an interesting solution to many small discrepancies one encounters when attempting to fit data with the $\Lambda \mathrm{CDM}$ model. These discrepancies include, the greater than expected number of superclusters in the SDSS [20, the extreme low density of matter within the voids 21, the number of satellite galaxies which is an order of magnitude smaller than predicted 23 among others. For sure, one can envisage other fixes for these

problems, such as, for instance, self-interacting dark matter (see e.g. Bento et al. 24] and references therein). Whatever solution, one concludes that evidence for a complex and interacting dark sector is mounting. It is then just logical to question whether a basic physical ingredient such as the EP may hold for this sector as well.

Acknowledgements The work of MLeD is supported by CSIC (Spain) under the contract JAEDoc072, with partial support from CICYT project FPA2006-05807, at the IFT, Universidad Autonoma de Madrid, Spain, and was also supported by FCT (Portugal) under the grant SFRH/BD/16630/2004, at the CFTC, Lisbon University, Portugal. The work of OB is partially supported by the FCT project POCI/FIS/56093/2004. The authors would like to thank Christopher Stubbs, Marc Kamionkowski and Rogério Rosenfeld for comments which prompt us to write this letter. We are particularly thankful to Zong Guo for permission to use a figure of Guo et al. [7.

\section{References}

1. D. Clowe et al., astro-ph/0608407

2. M.J. Jee et al., astro-ph/0705.2171.

3. O. Bertolami, F.G. Pedro and M. Le Delliou, Phys. Lett. B 654 (2007) 165.

4. C.W. Stubbs, Phys. Rev. Lett. 70 (1993) 119.

5. M. Kesden and M. Kamionkowski, Phys. Rev. Lett. 97 (2006) 131303; Phys. Rev. D. 74 (2006) 083007.

6. G.R. Farrar and R.A. Rosen, astro-ph/0610298

7. Z.-K. Guo, N. Ohta and S. Tsujikawa, astro-ph/0702015

8. See e.g. S. Schlamminger, K.-Y. Choi, T.A. Wagner, J.H. Gundlach and E.G. Adelberger, Phys. Rev. Lett. 100 (2008) 041101 and references therein.

9. O. Bertolami, J. Páramos and S.G. Turyshev, gr-qc/0602016.

10. M.C. Bento, O. Bertolami, A.A. Sen, and N.C. Santos, Phys. Rev. D 71 (2005) 063501; O. Bertolami and P. T. Silva, Mon. Not. Roy. Astron. Soc. 365 (2006) 1149; M.C. Bento, O. Bertolami, M. Rebouças and P.T. Silva, Phys. Rev. D 73 (2006) 043504.

11. A. Kamenshchik, U. Moschella and V. Pasquier, Phys. Lett. B 511 (2001) 265; N. Bilic, G.B. Tupper, R.D. Viollier, Phys.Lett. B 535 (2002) 17; M.C. Bento, O. Bertolami and A.A. Sen, Phys. Rev. D 66 (2002) 043507. 
12. L. Amendola, G.C. Campos and R. Rosenfeld, Phys. Rev. D 75 (2007) 083506.

13. L. Amendola, Phys. Rev. D 62 (2000) 043511.

14. Y. Wang and P. Mukherjee, Astrophys. J. 650 (2006) 1.

15. P. Astier et al., Astron. Astrophys. 447 (2006) 31

16. D.J. Eisenstein et al. [SDSS collaboration], Astrophys. J. 633 (2005) 560.

17. M.C. Bento, O. Bertolami and A.A. Sen, Phys. Rev. D 70 (2004) 083519.

18. E. Majerotto, D. Sapone and L. Amendola, astro-ph/0410543

19. V. Springel and G.R. Farrar, astro-ph/0703232

20. J. Einasto et al., astro-ph/0604539

21. G.R. Farrar and P.J. Peebles, Astrophys. J. 604 (2004) 1; A. Nusser et al., Phys. Rev. D 71 (2005) 083505.

22. O. Bertolami and J. Gárcia-Bellido, Int. J. Mod. Phys. D 5 (1996) 363; O. Bertolami, J.M. Mourão and J. Pérez-Mercader, Phys. Lett. B 311 (1993) 27.

23. B. Moore et al., Astrophys. J. Lett. 524 (1999) L19; A.A. Klypin, Astrophys. J. Lett. 522 (1999) L82.

24. M.C. Bento, O. Bertolami and R. Rosenfeld, Phys. Lett. B 518 (2001) 276. 